\title{
Studying children's social learning experimentally "in the wild"
}

\author{
EMMA FLYNN \\ Durham University, Durham, England \\ AND \\ Andrew Whiten \\ University of St Andrews, St Andrews, Scotland
}

\begin{abstract}
Diffusion studies are taking us a step closer to understanding social learning and cultural transmission in young children. The first half of this article presents a review that focuses on four main cultural issues addressed by diffusion studies: (1) horizontal transmission, including child-to-child learning; (2) learning in children's everyday environments ("in the wild"); (3) the experience of multiple demonstrations and attempts at mastering new tasks; and (4) the iterative process of learning across multiple cultural "generations." The second half of the article introduces an open-diffision experiment. After an initial asocial-learning phase in which children had the chance to discover two possible solutions to a puzzle box, the box was brought into the children's playgroup, thus allowing observational learning. Although variation of method use occurred in the asocial-learning phase, by the end of the second day of the open diffusion, the group had converged on a single method. The open-diffusion approach allowed the documentation of social interactions not seen in the dyadic studies typical of the field, including both coaction and scrounging, the significance of which for cultural transmission is discussed.
\end{abstract}

Young children are cultural creatures, using cultural tools (spoons vs. chopsticks) and cultural gestures (waving vs. rubbing noses), acquiring cultural norms such as stereotypes (Hill \& Flom, 2007), and, of course, acquiring one of the most critical cultural tools, language (see Sandler, Meir, Padden, \& Aronoff, 2005, for an example of the creation, transmission, and evolution of a less traditional communication system that uses gestures). Understanding a child's acquisition and transmission of these cultural behaviors is clearly an important scientific goal, but capturing such a complex phenomenon is difficult. We as humans live within a rich, culturally laden environment surrounded by cultural artifacts and behaviors, so naturalistic observation in this environment would appear to offer an opportunity to investigate and describe how behaviors are transmitted from one individual to another. However, although naturalistic observation can be extremely informative, a causal role for social learning is difficult to establish without controlled experimental intervention; the result, until quite recently, was more likely to be a descriptive account of the phenomenon, rather than a specific testing of hypotheses relating to the mechanisms underpinning behavioral change.

At the other extreme, numerous experimental studies have investigated specific hypotheses about the antecedents, consequences, mechanisms, and interrelations of different social contexts and cognitive skills in chil- dren's social learning. Such social learning is assumed by many authors to be a critical ability underpinning cultural transmission (Boyd \& Richerson, 1985; Plotkin, 2003; Richerson \& Boyd, 2005; Tomasello, 1999), although others have highlighted the complementary role of trialand-error learning in the transmission and development of cultural forms (Sterelny, 2006). We note, however, that the experimental work on social learning in children has almost exclusively used a dyadic design, in which a single child participant watches another individual (usually an adult) perform a behavior and then is given the opportunity to interact with the task apparatus, to discover whether he or she then will perform the behavior or variants of the behavior witnessed. Current areas of interest within this field include the role of selective social learning (i.e., whether a child copies means or goals; Carpenter, Akhtar, \& Tomasello, 1998; Carpenter, Call, \& Tomasello, 2005; Meltzoff, 1995), the role of ostensive pedagogy and eye gaze in children's interpretation of a task (Csibra \& Gergely, 2009), the underpinning cause of overimitation (Lyons, Young, \& Keil, 2007; McGuigan, Whiten, Flynn, $\&$ Horner, 2007), and how a model's identity and behavior affect a child's interpretation of a task (Nielsen, 2006; Wood, Kendal, \& Flynn, 2010).

Although such dyadic studies have been extremely informative, there are four main cultural issues that they typically fail to address. First, it is undeniable that the adults in 
a child's life are extremely influential in his or her cognitive and social development (e.g., theory of mind; Lewis, Freeman, Kyriadidou, Maridaki-Kassotaki, \& Berridge, 1996), but children may also learn from their peers; in this way, information may be transmitted horizontally (within generations), rather than vertically (between generations). Experimental work has shown that the identity of a partner influences an infant's behavior on a task; for example, infants as young as 3 months show a preference for female over male faces (Quinn et al., 2008). Model identity has specific theoretical import too, as highlighted, among other biases, by prestige bias, where observers copy individuals held in high, rather than low, esteem (Henrich \& Gil-White, 2001; Laland, 2004).

Second, although developmental psychologists are well trained in the practice of putting children at their ease in such a setting, it is undeniable that an experimental setting, with a single experimenter (a relative stranger, who often acts as the model) and a child participant, must, at the very least, be a specialized and even uncommon experience for a young, nursery-aged child. As such, it is possible that children may feel under special pressure in this strange situation; they may thus behave in a way that is different from how they would behave if faced with the same task in a more familiar environment, such as their home or a nursery group. Such a dynamic is again of theoretical import, since copy-if-uncertain (Laland, 2004) may be a sociallearning strategy used not only if one is uncertain about the task, but also if one is uncertain about the significance of the environment in which the task is presented.

Third, in dyadic studies, children often experience only one or two trials on a task. Such a constraint does not reflect a naturalistic setting in which children witness multiple demonstrations and have the opportunity for multiple attempts at a new task. Giving children the opportunity to witness multiple attempts in a study means that these attempts can be experimentally manipulated - for example, changing the identity of the demonstrator across trials or the accuracy of the demonstrated attempt. Likewise, allowing a child multiple attempts following demonstration means that change over trials can be examined, such as improvement in the efficiency of a method (ratcheting) or the potential for increased fidelity to a demonstration.

The fourth and most fundamental issue we wish to highlight is that culture is a much bigger phenomenon than the simple dyadic relations found in many social-learning studies; it is, in reality, a population-level phenomenon that involves the repeated transmission of information as it spreads across individuals, both within and between generations. It is therefore essential that social-learning experiments be extended to mimic the transmission of information and behavior within and between groups. Such studies with adults, using diffusion experiments, in which behavior is tracked within groups (a method described in full in the following section), have shown that changes in behavior may be small within a single dyadic transmission (adult to adult) but that, over generations of such episodes, the change can be significant. In the laboratory microsocieties created in these experiments, paper airplanes fly farther at the end of a series of generations than at the beginning of the series (Caldwell \& Millen, 2009, 2010), spaghetti towers get taller (Caldwell \& Millen, 2008), and an initially random language becomes more systematic and learnable (Kirby, Cornish, \& Smith, 2008). To investigate the larger phenomenon of culture, where the process of transmission is investigated beyond the dyadic setting and is extended to groups of individuals, we need a different method, one that allows for an investigation of change across cultural generations. Diffusion designs offer such a method.

\section{Experimental Studies of Culture}

Diffusion studies were first introduced by Bartlett (1932), who was interested in the recall of narratives (folktales and newspaper reports) along generations of individuals when each participant was presented with the recall produced by the previous participant. The approach has experienced a renaissance in recent times, such that Mesoudi and Whiten (2008) were able to review a varied corpus of 34 such studies with human adults. Similarly, researchers in animal social learning, with an interest in cultural transmission, have recognized the significance of the diffusion experiment and have developed it to test and study the spread of traditions in controlled conditions. A review by Whiten and Mesoudi (2008) identified 33 such studies in fish, birds, primates, and other mammals, from the first in 1972 up to 2008 (see also Reader \& Biro, 2010).

Since the pioneering work of Bartlett (1932), a number of alternative diffusion designs have been used (see Figure 1 for an illustration). First, diffusion chains follow an iterative dyadic design in which, after an initial, trained model begins the chain, the individual who learns from the initial model becomes the model for the next in the chain, and so on. The diffusion chain design offers a controlled method for examining transmission of behavior across individuals within groups, as the experimenter assigns participants to, and positions them within, the experimental chains. The diffusion chain design allows systematic comparisons to be made regarding factors that may affect transmission, such as sex, temperament, intelligence, and expertise. The second, replacement diffusion method is similar to the diffusion chain design but involves a group of individuals who work on a task during the same period, with the longest serving member of each group then being replaced by a new naive member after a certain number of task attempts. Such a design may represent a better approximation of some real-world contexts, such as staff turnover in a business, but it still incorporates a certain degree of experimental control by, again, allocating individuals to chains or positions in chains. A third design is open diffusion, where a model trained, or naturally proficient, in a behavior of interest is introduced, along with the task, to a group of novices. Such a design provides less experimental control, since participants choose when, or indeed whether, to attempt the task. The resulting transmission of behavior is thus potentially much messier than diffusion chains or replacement designs but represents a more realistic and, therefore, ecologically valid context in which to explore social learning and cultural transmission. 
A

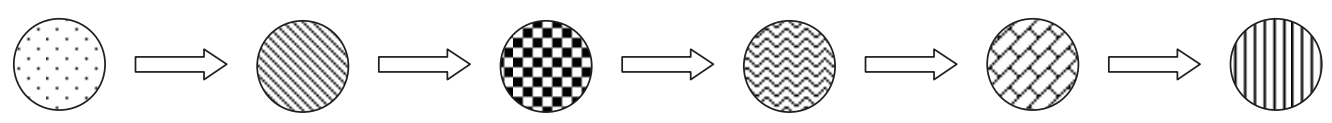

B
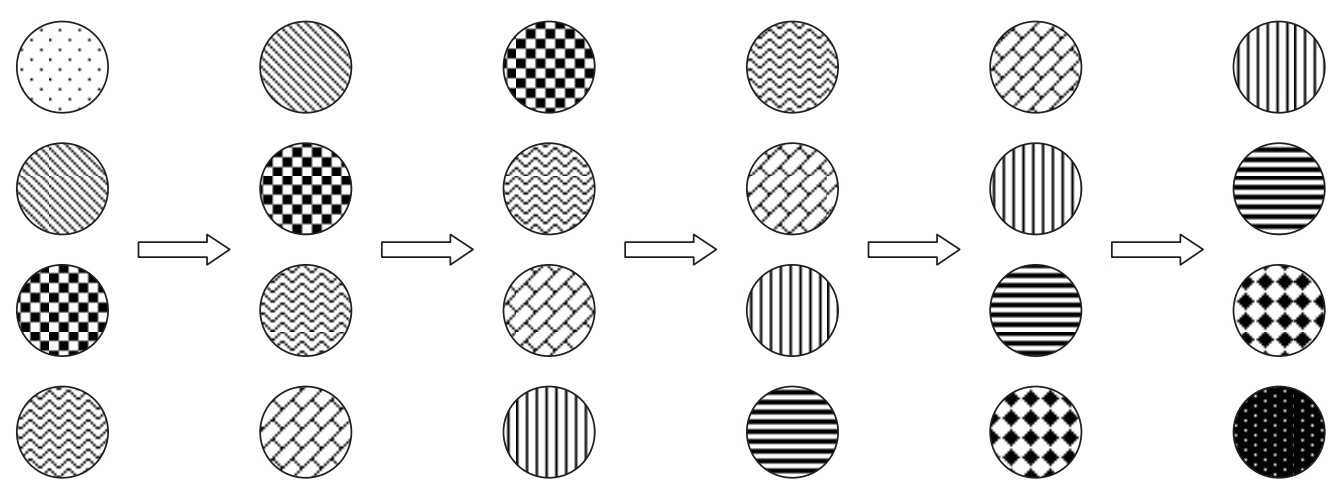

\section{C}<smiles></smiles>
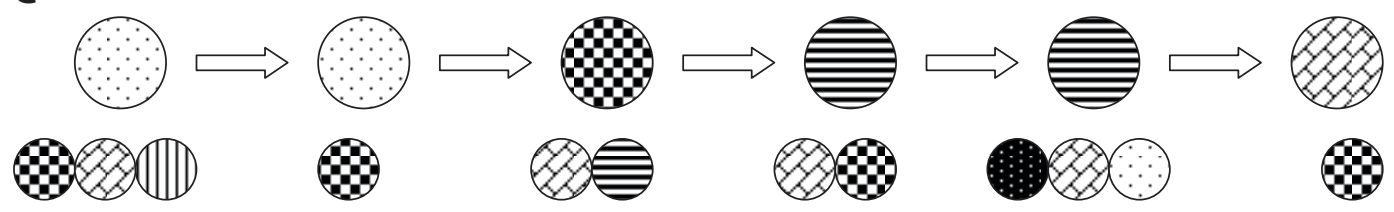

Figure 1. Diffusion designs. The pattern in each circle represents an individual's identity in diffusion chains (A), replacement chains (B), and open diffusion (C). In panel $C$, the larger circles represent individuals working on a task at one time, and the smaller circles represent those individuals who are observing the "actor's" behavior. These observers are free to attempt the task themselves and to come and go as they wish.

We can perhaps think of this as studying social learning experimentally with children "in the wild."

Mesoudi and Whiten (2008) and Whiten and Mesoudi (2008) have offered extensive reviews of the use of these diffusion designs with human adults and nonhuman animals, respectively. However, notably absent from these otherwise comprehensive reviews were diffusion studies in which the participants were children. The reason was simple: Scarcely any such studies had been published. Such studies have recently begun to proliferate, however, and below we review five of these child diffusion experiments. We then describe the results from a new study that used an open-diffusion design. The study investigated how information is spread when individuals in a group have an opportunity to acquire some personal information about the task before any social diffusion begins. We present these new data with specific reference to the methodological concerns and constraints that one needs to consider and adapt to when carrying out an open-diffusion study with children.

\section{Diffusion Studies With Young Children}

In this section, we review five studies in which diffusion chains were used to address theoretically interesting questions. All the studies have used a common methodology, aside from the diffusion chain design itself, and so, for economy, we outline the general method and rationale here. First, all the studies used the extraction of a reward from some kind of artificial fruit as the behavior of interest to be transmitted. Initially developed to study social learning of foraging techniques in nonhuman primates (Whiten, Custance, Gomez, Teixidor, \& Bard, 1996), artificial fruits are puzzle boxes that contain a reward that is trapped by single or multiple defenses that need to be removed before the reward can be retrieved, as in processing a real, complex fruit (Flynn \& Whiten, 2008b; Whiten et al., 1996). An observer watches a model extract the reward from the artificial fruit and is then presented with a rebaited artificial fruit that has all the defenses in place. A critical experimental point is that all the artificial fruits used in these diffusion studies are designed to permit at least two methods that allow extraction of the reward. For example, in one of the simplest forms, a reward may be held in place by a door and accessed by sliding the door either to the left (Method A) or to the right (Method B). The existence of these two methods allows the authors of these articles to use a three-group design that includes Methods A versus B, plus a no-model control group (Whiten, Horner, \& de Waal, 2005). A distinction can then be drawn about whether observers are copying the means used to achieve a goal (the specific method, A or B, used to extract the reward) and thus, in a broad sense, copying what they 
witnessed, or whether the observers have learned merely that the artificial fruit contains a reward, a goal that they might then achieve through their own means-that is, goal emulation (as in McGuigan et al., 2007). Since different chains of individuals are seeded with an initial model trained to use one of the two methods, the principal research questions are whether the alternative methods seeded at the beginning will be faithfully replicated along the respective chains or not and, in the latter case, whether the children will switch between methods or will consistently transfer to an alternative method.

In the no-model control group, participants are simply presented with the artificial fruit individually and are allowed to interact with it, without having witnessed a model's demonstration. This can provide two important pieces of information. First is the baseline level of success. Pilot work will ideally have generated not a task that is so easy that most children in the no-model control condition can extract the reward or so difficult that even children in the observational-learning condition cannot master it but, instead, a task that produces a level of success somewhere in between. The actual level of success without benefit of seeing a model is then established in the control condition, for comparison with the two conditions in which specific methods are seeded. Second, the control condition also provides an indication of any preexisting biases in relation to the alternative methods of solution possible. For example, when an artificial fruit in which a lever can be pushed or pulled to access a reward is presented, if all the children who are successful at the task during the no-model control condition pull the lever, this shows that children who push the lever in the observational-learning condition are more likely to have acquired this behavior through observational learning than through individual interaction with the task (a conclusion that may be validated through use of the option bias method highlighted in this issue by Kendal et al., 2010).

Horner, Whiten, Flynn, and de Waal (2006) completed the first diffusion chain study that had children as participants. The study made a direct comparison between chains of 3-year-old children and chains of chimpanzees, with both sets of chains begun by a model trained to either slide or lift a door on an artificial Doorian fruit to retrieve a reward. The results showed that, like the chimpanzees in this experiment, 3-year-olds were faithful to the method that was originally seeded, with the 8 children in the chain seeded with sliding consecutively sliding the door, and the 8 children in the chain seeded with lifting consecutively lifting the door, so that the last child in each chain did what the first child had done. In the nomodel control condition, $53 \%$ of the children were successful, 4 discovering lift and 4 discovering slide. These results underline the strength of the cultural transmission results, since both methods appear to be equally available to children of this age in their baseline behavior, so that the replication of the methods along the diffusion chains is particularly pertinent. Similarly for the chimpanzees, 3 of 6 control individuals opened the door, 2 discovering lifting and 1 discovering sliding. Horner et al. concluded that the exclusive use of just one of the available tech- niques in the diffusion chains may represent a form of canalization, whereby the potentially limitless exploration of a problem is, instead, focused around only a subset of behaviors that participants see performed by others, creating small-scale traditions.

Since Horner et al. (2006) had demonstrated that it was possible to run diffusion chains so effectively with young children, Flynn and Whiten (2008a) used this approach to investigate whether sex would affect children's social learning. We noted that Lonsdorf, Eberly, and Pusey (2004) had found that male juvenile chimpanzees learned through direct experience in playing at a termite mound, whereas females spent more time than did males watching their mothers fishing for termites and successfully fished for termites over a year earlier than males, a very marked sex difference. Accordingly, Flynn and Whiten (2008a) ran a diffusion experiment that contained male-only and female-only chains of children. It was hypothesized that if children behave like Lonsdorf et al.'s chimpanzees, girls would show stronger observational learning, with stronger fidelity to the method seeded, than would boys. The study also examined age effects, with chains containing only 3 -year-olds or only 5 -year-olds. We anticipated that there should be a difference in fidelity between 3-year-olds and 5 -year-olds, in line with advances in cognitive skills such as executive functioning (Flynn, 2007) and in sociocognitive skills such as theory of mind (Flynn, 2006). However, we argued that it was not possible to predict the direction of any such effect, since older children, relative to younger ones, might show stronger fidelity due to their greater attention and memory skills or, in contrast, might use their relatively sophisticated cognitive skills to be innovative and, so, be less beholden to what they had witnessed. To assess this, children were tested on an artificial fruit, the extractive-tool-use task (ETT; see Figure 2), which was a more complex task than the Doorian fruit (Horner et al., 2006). A counterpart of this task, designed to somewhat mimic termite fishing by wild chimpanzees, had been used earlier in open-diffusion experiments with this species (Whiten et al., 2007). For the children, the ETT was a plastic box that contained polystyrene beads that could be extracted using a tool. Two tools were available, since the two methods required alternative tools. The beads could be extracted using a stabbing method in which a stab tool with a pronged end could be inserted into a hole in the ceiling of the ETT box covered by a door and the beads could be stabbed and then extracted back through the hole. The alternative method used a slide tool, shaped like a ruler, that could be inserted through a slot at the base of the ETT box and then used to guide the beads to a bottomless chute at the opposite side of the ETT box.

The ETT presented a more complex task than did the Doorian box, since it required the use of a tool and a sequence of actions (pick up tool, open a door that covered either hole, insert tool, hold door open, maneuver beads, remove beads) to access the rewards. The difference in level of difficulty from the Doorian fruit used in Horner et al. (2006) could itself have produced different results, such as lesser fidelity across the chains. Interestingly and supporting this possibility, 4 children in the no-model 
A

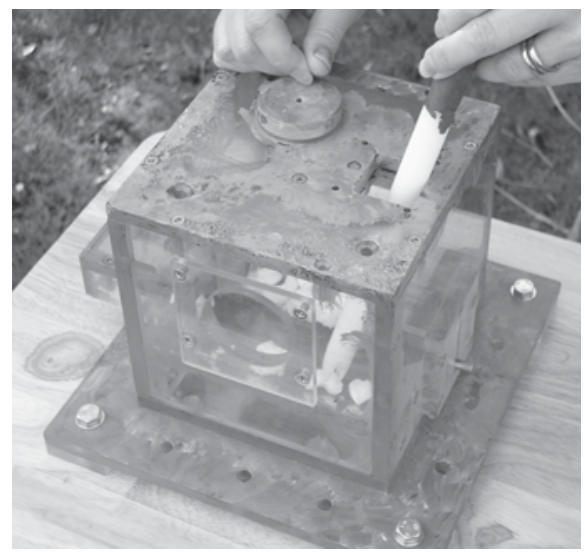

B

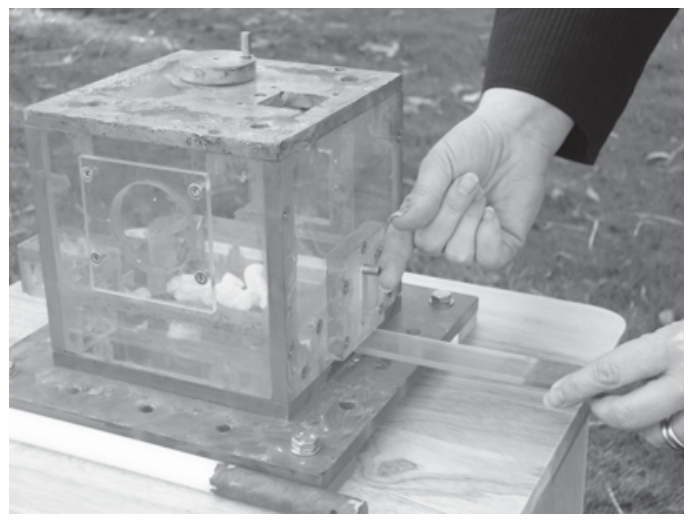

Figure 2. Extractive-tool-use task. (A) Stab method. (B) Slide method.

control condition found an alternative method for solving the ETT, since they inserted the slide tool into the hole in the ceiling and flicked the beads into the chute. Thus, the children in the observational-learning diffusion chains had not only the alternative methods designed by us (stab and slide) available to them, but also this third method that at least some children invented themselves. Nevertheless, as in Horner et al., the results showed strong fidelity in copying, with children who witnessed stab stabbing and children who witnessed slide sliding, even though sliding never occurred during independent exploration of the task in the no-model control condition (stab was performed by $15 / 47$ control children, with the other 28 children not extracting a bead). Interestingly, there was a single exception; a 3-year-old girl, who had witnessed the slide demonstration, tried to complete a stab during her first attempt. However, she was unsuccessful and demonstrated sliding to the next child. Nevertheless, her innovation hints that, occasionally, children may break away from the canalization process that characterizes most children's approaches, and this could possibly initiate some cultural change.

Overall, the results showed that both 3-and 5-year-olds were capable of transmitting a tool use method horizontally along cultural generations. Five-year-olds were more proficient than 3-year-olds at transmitting the technique faithfully across multiple generations, since 3-year-olds produced more unsuccessful attempts. Yet neither 3- nor 5 -year-olds attempted to innovate when they had observed another child complete the task using a particular technique, demonstrating strong cultural canalization. Contrary to expectations, girls showed poorer transmission than did boys, but this effect was found in just one of the four attempts, which was when they initially attempted the task in front of a naive peer. Girls were also less likely than boys to complete the task, even though they had witnessed a familiar peer having two attempts at completing the task. However, we believe that this result may be a function of the tool-based task used, since it may have motivated boys more; thus, in future research, girls may show stronger fidelity to more female-centered tasks.
Flynn (2008) and McGuigan and Graham (in press) used the diffusion chain design to address similar hypotheses relating to overimitation, a phenomenon identified by the copying of goal-irrelevant actions. Horner and Whiten (2005) investigated whether chimpanzees and 3-year-old children would copy visibly causally irrelevant actions demonstrated by an experimenter. To do this, they used a glass ceiling box (GCB; see Figure 3), a plastic box with a hole in the top that was defended by a bolt.

On one side of the GCB was a door that could be lifted or slid to reveal an opaque tube that contained a reward. The experimenter demonstrated a series of actions, some directed to the top hole and some directed to the door and the tube. Actions directed to the top (removing bolts, tapping into the hole) actually had no causal connection with retrieving the reward, for, in this case, the tool struck a partition (glass ceiling) lying above the tube containing

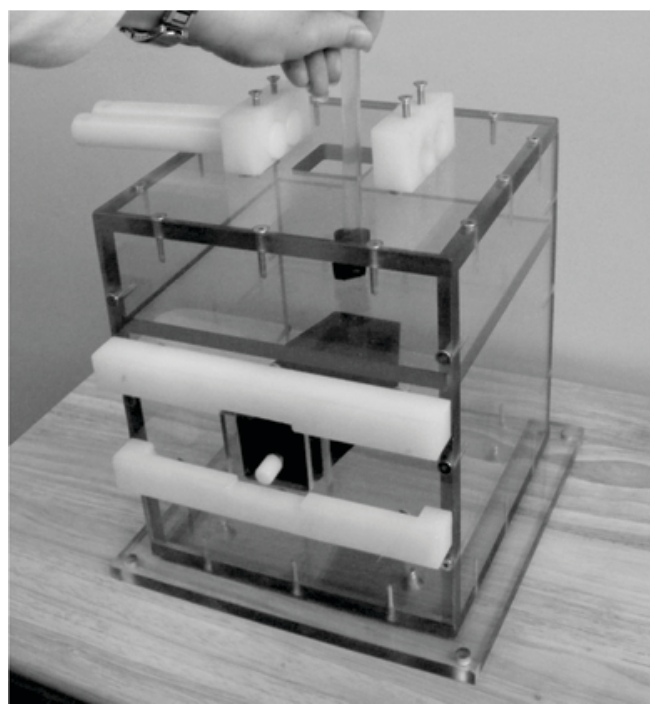

Figure 3. Transparent glass ceiling box with tool being tapped into the upper compartment. 
the reward, whereas actions directed to the door and the sloping tube were essential for task solution. Thus, the model demonstrated a mixture of causally irrelevant and relevant actions. Furthermore, the experimenter demonstrated these actions on either of two different versions of the GCB, one that was transparent, so that the casual irrelevance of the actions was visible, and one that was opaque, so that the irrelevance was hidden. Horner and Whiten (2005) presented 3-year-old children and young chimpanzees with both versions of the GCB in a counterbalanced order, some witnessing the demonstration with the transparent GCB first, others that with the opaque GCB. Chimpanzees showed an ability to discern that the actions directed to the top of the transparent GCB were irrelevant and tended not to reproduce them, but they tended to reproduce the relevant actions. They were able to transfer this knowledge to the opaque GCB if they began with the transparent GCB. However, those chimpanzees presented with the opaque GCB first copied the irrelevant actions, since the lack of causality of these actions could not be discerned. By contrast, the children did not show this pattern of behavior; they typically copied all actions, on both transparent and opaque GCBs. Flynn (2008) hypothesized that the children's indiscriminate copying may have been due to the authority of the adult model in this dyadic setting. If so, in diffusion chains with young children using the GCB, where the demonstrator is a peer rather than an adult experimenter, the irrelevant actions may be more likely to be filtered out. Flynn (2008) compared eight chains of children, containing either 2-year-olds or 3-yearolds, who were presented with either the opaque or the transparent GCB. An initial child model was trained to use both irrelevant and relevant actions. The results showed no significant difference across the eight chains based on either age or access to causal information, as represented by comparisons between the opaque and transparent GCBs. For all the chains, transmission of the irrelevant actions never reached the fifth and final child. Tapping into the upper compartment was never transmitted beyond the second child in each chain, and it was only in three of the eight chains that the second observer actually tapped. Removal of the bolts was slightly more robust, but again this never occurred by the end of any chain, persisting only to the second child in two of the eight chains and the fourth child in two other chains. Flynn (2008) suggested that the slightly higher replication of the removal of the bolts may have been a primacy effect, since bolt removal was the first action in the sequence of actions. Unlike the irrelevant actions, the method used to perform the causally relevant action of opening the door (lift vs. slide) in order to retrieve the reward showed strong fidelity, with $87 \%$ of the children copying the witnessed method on their first attempt and $77 \%$ or more of the children replicating this method on their subsequent attempts, in line with the findings in Horner et al. (2006). Flynn (2008) concluded that 2- and 3-year-old children are capable of a form of cultural evolution - that is, cumulative culture - in which they parse out irrelevant actions in a sequence, using the behavior they had witnessed to achieve a goal but making it increasingly efficient as the microculture developed.
McGuigan and Graham (in press) independently used a quite similar design, employing diffusion chains with the GCB, but they used single chains of either 3-year-olds or 5 -year-olds with either the opaque or the transparent GCB, resulting in four chains, as opposed to the eight used by Flynn (2008). Whereas in Flynn (2008), children had four attempts (two initial attempts and two demonstration attempts in front of the next child in the chain), the children in McGuigan and Graham had just two attempts (one initial and one demonstration). For the 5-year-olds who were presented with the transparent GCB, the first child after the model performed all the relevant and irrelevant actions; however, all the subsequent children in the chain omitted the irrelevant bolt removals and stabbing in the top hole. Five-year-olds who were presented with the opaque box came to omit the tool insertions into the upper compartment but showed fidelity along the chains with regard to the bolt removals (although by the fifth child in the chain, only one bolt had been removed). Thus, 5 -yearolds appear to be more sensitive to the causal information they have available to them, since they show fidelity of transmission of redundant information when the causal information is ambiguous; but when presented with clearer causal relations, 5-year-olds selectively acquire only relevant behaviors. This contrasts rather strikingly with dyadic experiments with the same task, which showed that 5 -year-olds persistently overimitate an adult model, even under conditions in which 3-year-olds do not (McGuigan et al., 2007).

When considering the fidelity of the 3-year-olds in McGuigan and Graham (in press), direct comparisons can be made with Flynn (2008). Surprisingly different results were obtained. Whereas Flynn (2008) found little transmission of irrelevant actions, regardless of the opaque/ transparent condition, McGuigan and Graham found strong transmission. As with Flynn (2008), McGuigan and Graham found that tapping in the top hole dropped out very quickly, but the transmission of the bolt removals was persistent, with children along the chain even using the same method that had been used to remove the bolts. Flynn (2008) did find that the bolt removal was more persistent than the tapping, but as was noted above, it occurred in only four of the eight chains and disappeared after the second child in two of the chains and after the fourth in the other two chains. Finally, whereas Flynn (2008) found that over $77 \%$ of the children were faithful to the method used to open the door to the reward, McGuigan and Graham reported weak fidelity to the method used to open the door. The contrasting results may, in part, have been due to the fact that in Flynn (2008), children received two demonstrations, whereas in McGuigan and Graham, they received only one. Although this may explain the fidelity to the door opening in Flynn (2008), it provides no explanation for why the transmission of irrelevant actions was strong in McGuigan and Graham, where fewer demonstrations were given. One would expect that more trials would equate to a higher perceived level of intentionality of action on the part of the model, yet when these two studies were contrasted using the most obvious methodological difference, more demonstrations did not equate to 
stronger transmission of irrelevant actions. The disparity in these results remains somewhat mysterious and begs further investigation.

Finally, Hopper, Flynn, Wood, and Whiten (2010) completed a diffusion chain study using another tool use artificial fruit task, the panpipes (Hopper et al., 2007; Whiten et al., 2005). In order to retrieve a reward (a capsule containing a sticker) in this task, a tool was used to lift (Method A) or push (Method B) an obstruction. Hopper et al. (2010) found that the method demonstrated was transmitted impressively along 20 generations, twice as many as has been used previously. Cultural transmission is a long-term phenomenon, and so it is extremely informative and important that the microsocieties produced in these diffusion studies reflect this long-term, multiplegeneration transmission.

The small initial corpus of child diffusion studies reviewed above shows that the diffusion chain design offers an informative method with which to examine social learning and the transmission of behavior across groups of young children, thus helping to build an experimental science of culture that extends the existing primarily dyadic social-learning literature. By culture, we refer to the definition in Richerson and Boyd (2005): "Culture is information capable of affecting individuals' behavior that they acquire from other members of their species through teaching, imitation, and other forms of social transmission" (p. 5). The results of these studies have shown that children as young as 2 years are capable of both transmission and refinement of a culturally transmitted technique, to make it more efficient (Flynn, 2008). In general, young children's actions become strongly canalized; once they have observed another individual complete a task, numerous options available to them in principle appear to become closed off, and children replicate what they have witnessed another individual do (Flynn \& Whiten, 2008a; Hopper et al., 2010; Horner et al., 2006). Flynn and Whiten (2008a) showed that diffusion chains additionally allow an investigation of the different theories relating to social learning. However, all five studies reviewed here used a diffusion chain design, which is only one of the three main diffusion designs occurring in the literature more generally, the others being the replacement method and open diffusion.

In the second half of this article, we introduce a study that used an open-diffusion design to investigate the importance of personal and social information for children's subsequent behavior. The data reported here are from a control condition that was part of a larger study (unpublished data), in which two individual models were trained to use either of the two alternative methods to open a new artificial fruit; once trained, the models and the task were introduced to each of the playgroups that the model attended. In the seeded conditions, which are not presented here, we witnessed much social learning, with behaviors being spread from the original seeding. In the control condition presented here, in which no method was experimentally seeded by a trained model but diffusion was still possible on the basis of the children's own innovations, we witnessed fewer signs of social learning than in the condi- tions in which a method was experimentally seeded. However, some extremely interesting and unexpected social phenomena occurred - notably, in the social interactions over the task and the effects of social dominance. We believe that a discussion of these in the light of the methodological demands of an open-diffusion design will provide researchers interested in adopting such an approach with a rounded understanding of this methodology.

\section{Children's Use of Personal and Social Information When "Foraging" for Rewards: An Open-Diffusion Study}

The primary issue investigated within the current data set was whether children continue to use previously acquired personal information to solve a task when faced with social information from multiple sources in a natural, open-diffusion setting, or whether they switch to alternative, socially acquired solutions. It may be that personal information, which has previously proved correct, will dominate a child's subsequent behavior, resulting in no change in the method he or she uses to extract a reward once in an open-diffusion setting. Alternatively, children may change the method they use, on the basis of what they witness during the open diffusion. If change does occur, the motivations facilitating the change may be multiple. For example, children whose asocially learned method is distinct from the majority of behavior they witness within the open diffusion setting may choose to adopt the group's method, possibly exhibiting conformity (Boyd \& Richerson, 1985). Alternatively, different methods may be demonstrated by dominant or popular children, and children may choose to adopt these methods, in line with prestige biases (Henrich \& GilWhite, 2001) or in favor of methods used by friends, thus demonstrating a copy-friends strategy (Laland, 2004). Similar questions could also be asked of children who had been unsuccessful at retrieving the reward during an initial asocial-learning phase but later acquired social information leading to success (see Franz \& Nunn, 2010, for an alternative method of assessing these types of questions in free-living populations).

The study had two stages: an initial asocial-learning stage, in which children were presented individually with an artificial fruit (the serial box [SB]; see Figure 4) with no model present and no goal-directed instruction. This phase allowed the children to acquire information about the task, such as how possibly to extract the reward or, indeed, what would not allow extraction of the reward. It also established whether one of the two methods to extract the reward was more salient. After this asocial-learning phase, the SB was placed into the children's usual playgroup in an area for a period of time during which it was free for all the children to access it. During the open-diffusion phase, we videotaped and later coded which children were manipulating the SB, whether they were successful at extracting the reward, and, if so, what method they used. This provided an ordered sequential record of all the children's attempts and successes at the task. Video records of the open-diffusion phase also allowed an analysis of which children witnessed these attempts and successes. 


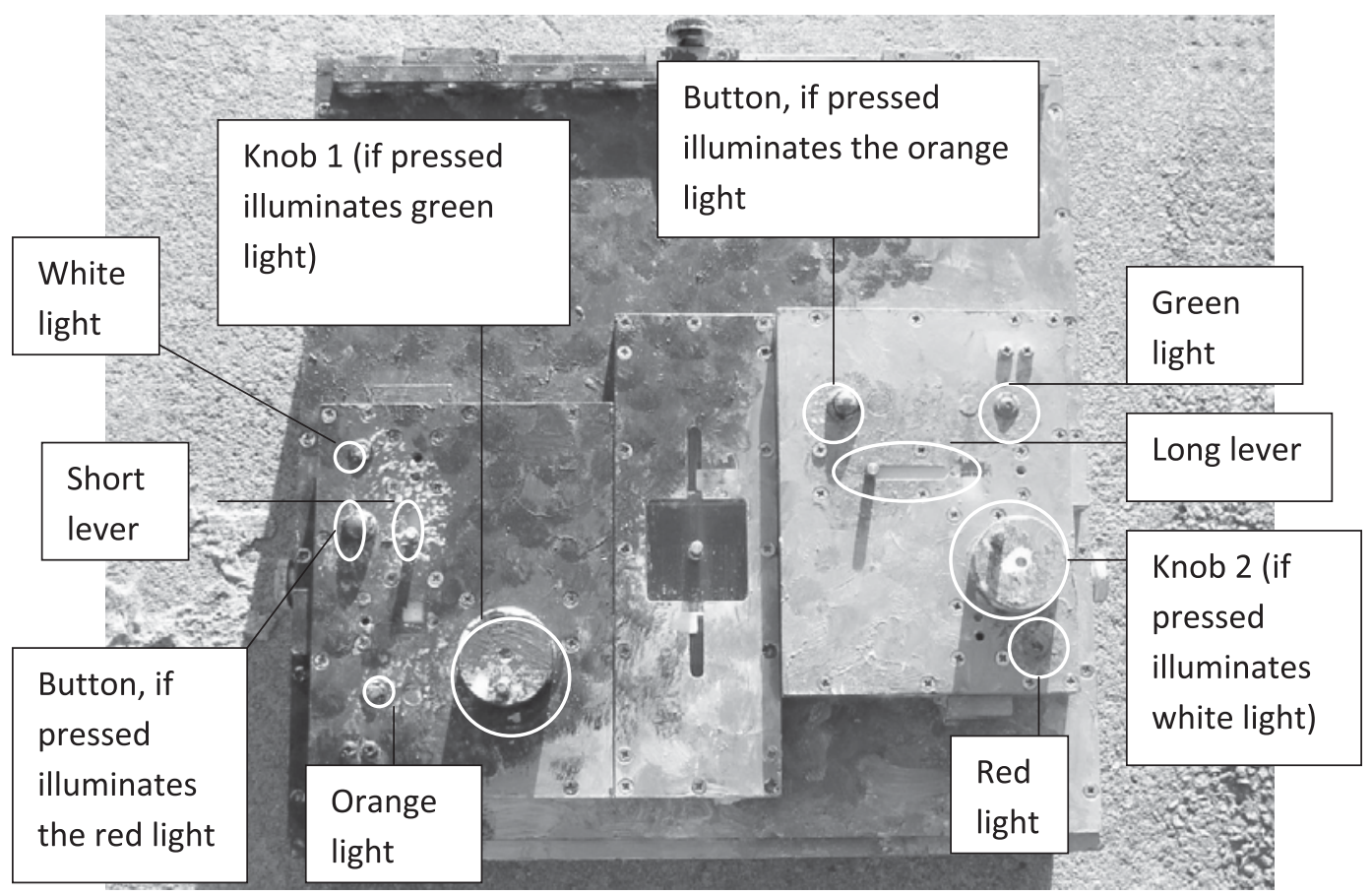

\begin{abstract}
Figure 4. Serial box (SB). The door at the center of the roof could be opened to reveal a compartment containing a reward; the compartment was part of a larger wheel of 12 compartments (each containing a reward) that could be moved by remote control. The door was held in place by a number of defenses and was released through two methods: Method A (push Lever A, then turn Knob 1) and Method B (turn Knob 2, and then push Lever B). The roof of the SB also contained two buttons, one on each side. Each button illuminated a different colored light (orange or red). A further light on each side could be illuminated by pressing each of the knobs (Knob 1 illuminated the green light, and the pink knob illuminated the white light).
\end{abstract}

The SB was a plastic box $(42 \times 40 \times 12 \mathrm{~cm}$ high $)$, which was painted so that its internal workings could not be seen, except for a clear, colorless plastic door $(5 \times 5 \mathrm{~cm})$ in the center of the upper surface, which allowed access to a compartment containing a sticker. This compartment was illuminated, and so, the desirable sticker was clearly visible. The door was held in place by a number of defenses, which could be released by using one of two methods: Method A (push Lever A, then turn Knob 1) resulted in the door's opening by sliding it toward oneself (if one was seated facing the SB, as in Figure 4); Method B (turn Knob 2 and then push Lever B) allowed the door to be opened by sliding it away from oneself. The two methods were designed to be equivalent in effort, ease, and success. Thus, unlike previous artificial fruit tasks-for example, the ETT (Flynn \& Whiten, 2008a) — we expected that there would be no predisposition to use one particular method over the other. Two buttons sat on top of the SB, and when one button was pressed, a red light was illuminated on the surface of the box, whereas pressing the other illuminated an orange light. Knobs 1 and 2 could also be pressed down, resulting in green and white lights, respectively, being illuminated. These light-related actions in fact had no causal role in opening the door (unlike the essential lever and knob operations) and were designed to be potential distractors with scope for testing overimitation. Inside the SB was a wheel with 12 compartments, each containing a sticker. The experimenter could rotate the wheel, so that a new sticker appeared under the door, through use of a remote control device $(5 \times 3 \mathrm{~cm}$, small enough to be discretely held in the palm of one's hand). This rebaiting automatically reestablished the defenses and extinguished the lights; thus, to gain access to the new compartment, Method A or B had to be implemented again.

The participants were 22 children who attended a playgroup in the east of Scotland (mean age $=3$ years 6 months, $S D=6$ months; range, 2 years 11 months to 4 years 2 months; 10 males). During the initial, individual asocial phase, the children were brought into a quiet but familiar room in the playgroup location and were told that "lots of boys and girls have had a go and now it is your turn," as the experimenter pointed to the SB. The children were allowed to interact with the SB until they successfully extracted the sticker; until they refused to continue after a prompt that included "what do you think you do now?" "you can touch it as much as you like, you can't break it," and "you're doing really well, what do you think you do next?"; or until 8 min had elapsed. Eleven children (50\% of the whole group) were successful, $6(55 \%$ of the successful children) using Method A and 4 (36\% of the successful children) using Method B. One child completed both methods before she pushed open the reward door. This supported our proposal that the two methods were roughly equivalent in their likelihood of spontaneous discovery. Seven of the 11 successful children (64\%) did not need a prompt to be successful; the remaining $4(36 \%)$ 
were successful after a prompt. It took the unprompted children a mean of $2 \mathrm{~min} 13 \mathrm{sec}$ to be successful (range, $57 \mathrm{sec}$ to $4 \mathrm{~min} 20 \mathrm{sec}$ ); those who were prompted were successful in $5 \mathrm{~min} 44 \mathrm{sec}$ (range, $2 \mathrm{~min} 40 \mathrm{sec}$ to $7 \mathrm{~min}$ $40 \mathrm{sec}$ ), therefore providing an overall mean success time of $3 \mathrm{~min} 29 \mathrm{sec}$. The mean time spent interacting with the task for the children who were not successful was 5 min $29 \mathrm{sec}$ (range, $2 \mathrm{~min} 27 \mathrm{sec}$ to $8 \mathrm{~min} 18 \mathrm{sec}$ ). There was no difference between the children who were successful at extracting the reward and children who were not successful according to age [mean (no success) $=43$ months, mean $($ success $)=41$ months; $t(20)=0.69, p=.49$ ] or sex [male:female ratio (no success) $=5: 6$, (success) $=6: 5$; $t(20)=0.41, p=.69]$. Children who retrieved the sticker were allowed to keep it, and children who were unsuccessful were given a sticker at the end of their participation.

The day after the asocial-learning phase, the opendiffusion phase began. The SB was placed in an area of the nursery that was accessible to all the children during a time when all the children were free to attempt it. This was done for 2 days for an hour on each day. The behavior demonstrated over the 2 days is illustrated in Figure 5.

There were 91 successful extractions of the reward from the SB, 30 using Method A (33\%) and 60 using Method B (67\%). Although all 22 children from the first phase had access to the SB, only 12 (55\% of the total) attempted the task during the open-diffusion phase. Of these, 6 had been successful and 6 had been unsuccessful during the asocial-learning phase. Six children who had been successful during the asocial-learning phase were successful during the open-diffusion phase. Four used the same method as that acquired during the asocial-learning phase for their first success in the open-diffusion phase. However, 2 children (E.C. and L.R.) changed to Method B on their first success and produced a successful extraction by undertaking a collaborative coaction, with each of them completing one element of the method (see Figure 5, Bout 1). Five of the 6 children who had been successful in the asocial-learning phase used both methods during the open-diffusion phase; the other child (G.F.) produced only one success during the open-diffusion phase. Two of these 5 children (E.C. and L.R.) produced a new method through collaboration (as described previously); the other 3 (E.M., G.M., and H.L.) all showed evidence of adopting a new method after having witnessed it being used by another child (as illustrated by the arrows in Figure 5). A further 6 children who had been unsuccessful during the asocial-learning phase attempted the SB during the open-diffusion phase; 3 of these (P.G., H.S., and M.C.) successfully retrieved the reward from the SB during the open-diffusion phase. P.G. replicated what he had previously witnessed during the open-diffusion phase, and H.S. and M.C. participated in separate collaborative attempts (see Figure 5, Bouts 8 and 10). Of the 9 children who were successful during the open-diffusion phase, 4 used both methods, 3 were faithful to a single method (all used Method B), and 2 produced only one attempt.

One key finding across the 2 days was that during the first day, children used a mixture of methods to achieve their successes; however, by the second day, all the children's successes were achieved using the same method, B. This convergence suggests some degree of social conformity, since the initial, asocial-learning phase showed that the two methods were within the group's repertoire at similar levels (A was, in fact, slightly more common than B), although caution is required, since 11 asocial-learning controls cannot unequivocally allow one to determine that there was no bias in the use of Method B over A. But the results suggest that there was not a trend toward the use of Method B over Method A in the open-diffusion phase simply because it was the children's predisposed method, since, of those children who responded in the opendiffusion phase, there was no prior asocially learned bias for Method B over A. Indeed, the swing to the Method B monoculture on Day 2 was to the method that had been most commonly seen (67\% of cases) on Day 1 . It is not possible to further probe these events statistically, insofar as the number of children working on the SB, and particularly those who changed their method, was too small.

A second noteworthy finding was the variation in behavior. For example, 11 children learned asocially, but 11 did not. Then 5 of those who learned asocially also learned socially, whereas 6 did not. Five children who learned asocially did not try again with the task, whereas 1 did and kept the same method, although he made only one attempt. Five others chose to change method from that learned through asocial learning to that witnessed during the open-diffusion phase. Whether a clearer pattern would have emerged with a larger sample or more time is unknown, but the data here suggest that cultural learning in such an everyday context can be both flexible and varied.

The critical difference between this study and our other open-diffusion studies (Whiten \& Flynn, in press) and, indeed, other diffusion chain and dyadic studies was that this study included an initial asocial phase, in which the children had a chance to acquire some information about the task. During this initial phase, $50 \%$ of the children were able to successfully remove the sticker, using a relatively balanced mixture of Method A and Method B. Thus, it would appear that the SB was a good task to use within an open diffusion, insofar as a number of participants were not initially successful at the task but there were also models who had been successful, from whom the unsuccessful children could learn. However, such extensive learning did not appear to happen; instead, only $55 \%$ of the children from the playgroup attempted the task in the opendiffusion phase. We hypothesize that one of two things may have happened. Children who had been successful in the asocial phase may have had their interest in the SB satiated; that is, having been successful they were not as motivated to acquire the sticker reward in the open-diffusion phase. Similarly, for children who had been unsuccessful on the SB during the asocial phase, their motivation may have been reduced, since they had attempted the task and had become frustrated and, essentially, had learned that they "couldn't do it."

An additional factor was that one child, E.M., dominated attempts on the task, producing $65 \%$ of the total ex- 


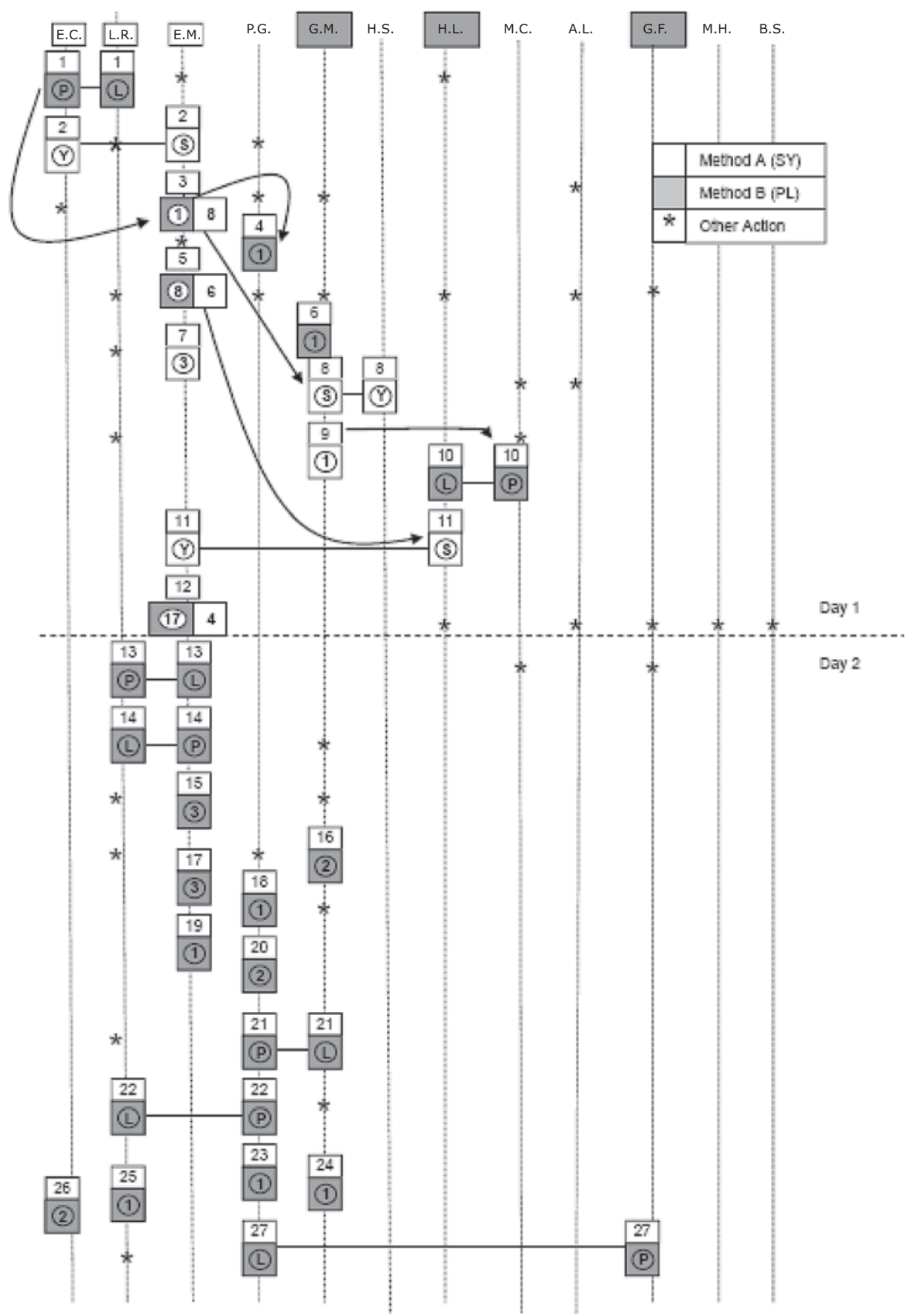

Figure 5. Open diffusion, over 2 days, with the serial box (SB). The columns represent the order of children's successes at extracting the reward during the open diffusion. The initials in rectangles across the top are for those children who were successful in the asocial phase; white rectangles represent use of Method A, and dark rectangles represent use of Method B. No rectangle means that the child was not successful in the asocial phase. Rows represent successful bouts, sometimes using different methods to complete a series of successful extractions within the same bout. Arrows represent witnessing and adopting a new method, for either a child who had never been successful or a previously successful child. Some attempts were joint attempts, represented by joining horizontal lines, with 2 children completing different parts of the extraction (P, turn pink knob; $Y$, turn yellow knob; L, push long lever; $S$, push short lever). An asterisk represents a child's interaction with the SB during another child's successful bout; these interactions included wiggling the door, attempting to turn an already turned knob, or pushing an already pushed lever and/or switching on lights. There were seven further bouts on the SB by M.C. and E.C., but these all used Method B. 
tractions. However, this does not fully explain the lack of participation of the other children, since there were long periods in which either E.M. was not working on the task and other children were or the task was free for any child to attempt it.

Future work might ameliorate the lack of motivation we inferred to be due to satiation or frustration by using a higher value, more desirable, and thus more motivating reward, whether from the beginning or from the start of the open-diffusion phase. However, it should be noted that stickers similar to those used in the present task have been used in our other open-diffusion studies, which have shown a high level of social learning (Whiten \& Flynn, in press). It may simply be that for children of the age we studied, the prime motivation for social learning about relevant manipulative techniques relies typically on the child's not already having a successful technique in his/ her repertoire, which in the present study, we undercut through the initial individual-learning phase. Having said this, for those children who did participate, the shift to the monoculture of Day 2 suggests a substantial effect of social learning and conformity.

An interesting and unexpected phenomenon was that the children sometimes worked on the task together (e.g., Figure 5 illustrates 10 incidents of coaction), and moreover, this also involved a degree of scrounging, where children who had not removed defenses nevertheless extracted the reward. The defenses and door to the reward were spread across the top of the SB (Figure 4), such that the children could manipulate the door (either by quickly opening the door or by sitting with their hand on the door) while another child was manipulating the defenses and releasing the door. On $40 \%$ of the extractions, a child obtained a reward when they had not released the defenses, either because they essentially "stole" the reward (i.e., got to the reward before the child who had removed the defenses [14\%]) or because the reward was given to them or they were invited to extract the reward by the child who had the removed the defenses (26\%). We can describe the different roles children take in such an open-diffusion task as enablers (or producers who remove the defenses), active scroungers (who take hold of a door to acquire a reward, although not having removed the defenses), or passive scroungers (waiting to be given a reward or invited to extract a reward).

These findings are of interest in relation to a significant literature on the relation between scrounging and social learning in the animal behavior literature. This relates to a long-standing distinction in behavioral ecology between producers, who actively gain food items, and scroungers, who manage merely to take resulting scraps or even steal whole items (Barnard \& Sibly, 1981). However, there are contrary findings on whether scrounging inhibits or facilitates social learning. Several studies have indicated the former, shown in the course of experimental studies of foraging pigeons (Giraldeau \& Lefebvre, 1987; Giraldeau \& Templeton, 1991). However, other experiments have shown scrounging to facilitate social learning-for example, in marmosets (Caldwell \& Whiten, 2003) and meerkats (Thornton \& Malapert, 2009), the latter even concluding that "scrounging appeared to be the primary driver of technique acquisition" (p. 255). Reasons for the different positive and negative results have not been resolved. It is typically assumed that the negative effects arise from consistent scroungers' simply not needing to learn, so long as they can maintain their parasitic success. The positive effects may arise from the intimacy with the model and the task that scrounging can facilitate, with what has been learned revealed only when the scrounger lacks a tolerant producer to exploit and must deal with the task itself. Indeed, it would be interesting to test whether scroungers in the studies reporting negative results would show positive results where opportunities for scrounging cease. Against the background of these comparative studies, it would be of interest to generate a statistically larger sample of scrounging than that uncovered in this first exploratory study and examine whether such behavior does, indeed, inhibit or facilitate learning, both in the producescrounger context (predicted negative effect) and where scrounging opportunities no longer exist (predicted positive effect). We note that scrounging is a phenomenon discovered only in the open-diffusion design; in other experimental designs, observers are explicitly not allowed to interact with the task at the same time as the demonstrator, and so, scrounging effects will not be seen. Using open diffusion allows the study of this natural phenomenon.

However, such studies should also take account of the differences in the tone of such interactions, which we have distinguished as active versus passive scrounging. The key point is that although some scrounging appears essentially competitive (corresponding to some of the animal behavior cases), other cases involve interactions more appropriately described as cooperative. Indeed, more broadly, social learning in the context of joint, even collaborative, interactions (Tomasello, Kruger, \& Ratner, 1993) between children working together on a task like the SB should be a point of attention in future studies.

Finally, it is worth reflecting on the methodological demands of undertaking an open-diffusion design with young children, some of which have been highlighted by this study and some of which have not. An initial question relates to why people have not attempted open-diffusion studies with children, especially since the corpus of opendiffusion studies with nonhuman animals is steadily growing and diffusion chains with young children have been shown to be very informative. Obviously, open-diffusion studies are challenging to undertake, since they require intensive examination of a process of change within a group of individuals. But this is true of open-diffusion studies with both humans and nonhumans. Yet carrying out open diffusion with humans, especially children, has added difficulties. First, informed consent must be given by parents and playgroup staff, and children must also be willing to participate. A critical point of open diffusion is that it is naturalistic and occurs in a child's usual playgroup area ("in the wild"); thus, the ideal situation is to get all playgroup parents and children to consent, so that the whole microsociety can be studied as one coherent and natural phenomenon. Even if one or two children decline to participate, this can change the dynamics of the environment 
significantly. Such a problem is not usual with nonhuman animals, since although an individual may decide not to participate, that individual is not actively removed from the testing session by the experimenter. Second, children show forms of social learning that are rare, if not nonexistent, in nonhuman animals. For example, as has been seen in this study, children may cooperate actively and intentionally with one another. Or, as has been seen in many other studies (e.g., Ashley \& Tomasello, 1998; Whiten \& Flynn, in press), they may teach one another, using direct verbal instructions as to how to solve a task. These alternative forms of social learning mean that not only does physical behavior need to be coded, but so does verbal behavior, which results in a complex teasing apart of the learning processes. On the other hand, it is undeniable that open-diffusion studies with young children are extremely informative in establishing how children learn in their usual environment, providing important information about how behavior is spread through a group. Finally, methodologically, using children can be very beneficial, since, once recruited, larger samples of these "cultural magnets" (Flynn, 2008) can be used, and the behavior in multiple, large groups can be compared to address specific theoretical questions, something that is not always possible with nonhuman species.

\section{Conclusions and Future Avenues}

We hope that we have convinced readers that it is possible to investigate children's social learning "in the wild," using experimental techniques that allow specific hypotheses to be addressed. Obviously, the different diffusion methods provide different levels of experimental control and accommodate or allow different levels of "wildness"; but it is clear that even with the most controlled of the diffusion methods, diffusion chains, we see a significant shift toward ecological validity, as compared with the usual dyadic experimental setting, since children learn from other, familiar children, alone or within groups, often in their usual playgroup. Now is a fruitful time to use diffusion methods to investigate children's social learning and the larger phenomenon of culture. Such investigation will need to include such questions as those sketched at the beginning of this article. For example, what is the role of selective learning (whether one copies means or goals, for instance)? Do we see fidelity to means across all tasks or just the artificial fruits that we use within our research, and is fidelity to means lessened in an open-diffusion versus a dyadic setting? Do individual differences in the ostensive cues that children provide in these natural settings affect the fidelity with which others copy them? If a model within an open-diffusion setting is trained to use irrelevant actions on a task, do these irrelevant actions get copied? The findings of Flynn (2008) would suggest not, but perhaps there are individual differences in the likelihood of whether a child will copy such irrelevant actions. Finally, to date and to our knowledge, there have been no replacement diffusion studies with young children. Such a method may offer great opportunities to explore such questions as how the makeup of different groups, whether based on sex mix, age mix, task expertise, or temperamental/personality factors, affects the cultural transmission of behavior across groups.

\section{AUTHOR NOTE}

This study was supported by a grant from the Economic and Social Research Council (RES000230603) to A.W., who was also supported by a Leverhulme Trust Major Research Grant and a Royal Society Leverhulme Trust Senior Research Fellowship. We thank the editors of this special issue for inviting us to contribute to an exciting and informative volume. We are grateful to Lara Wood and Rachel Kendal for comments on an earlier version of the manuscript. Thanks also go to all the children, parents, and staff who assisted with this work. Correspondence concerning this article should be addressed to E. Flynn, Department of Psychology, Durham University, Science Laboratories, Durham DH1 3LE, England (e-mail: e.g.flynn@durham.ac.uk).

\section{REFERENCES}

Ashley, J., \& Tomasello, M. (1998). Cooperative problem-solving and teaching in preschoolers. Social Development, 7, 143-163.

BARNARD, C. J., \& Sibly, R. M. (1981). Producers and scroungers-A general model and its application to captive flocks of house sparrows. Animal Behaviour, 29, 543-550.

Bartlett, F. C. (1932). Remembering: A study in experimental and social psychology. Cambridge: Cambridge University Press.

Boyd, R., \& Richerson, P. (1985). Culture and the evolutionary process. Chicago: University of Chicago Press.

Caldwell, C. A., \& Millen, A. E. (2008). Experimental models for testing hypotheses about cumulative cultural evolution. Evolution \& Human Behavior, 29, 165-171. doi:10.1098/rstb.2008.0133

Caldwell, C. A., \& Millen, A. E. (2009). Social learning mechanisms and cumulative cultural evolution: Is imitation necessary? Psychological Science, 20, 1478-1483.

Caldwell, C. A., \& Millen, A. E. (2010). Human cumulative culture in the laboratory: Effects of (micro) population size. Learning \& Behavior, 38, 310-318.

Caldwell, C. A., \& Whiten, A. (2003). Scrounging facilitates social learning in common marmosets, Callithrix jacchus. Animal Behaviour, 65, 1085-1092.

Carpenter, M., Akhtar, N., \& Tomasello, M. (1998). Fourteenthrough 18-month-old infants differentially imitate intentional and accidental actions. Infant Behavior \& Development, 21, 315-330. doi:10.1016/S0163-6383(98)90009-1

Carpenter, M., Call, J., \& Tomasello, M. (2005). Twelve- and 18 -month-olds copy actions in terms of goals. Developmental Science, 8, F13-F20. doi:10.1111/j.1467-7687.2004.00385.x

Csibra, G., \& Gergely, G. (2009). Natural pedagogy. Trends in Cognitive Sciences, 13, 148-153.

FLYNN, E. (2006). A microgenetic investigation of stability and continuity in theory of mind development. British Journal of Developmental Psychology, 24, 631-654. doi:10.1348/026151005X57422

FLYNN, E. (2007). The role of inhibitory control in false belief understanding. Infant \& Child Development, 16, 53-69. doi:10.1002/ icd. 500

FLYNN, E. (2008). Investigating children as cultural magnets: Do young children transmit redundant information along diffusion chains? Philosophical Transactions of the Royal Society B, 363, 3541-3551. doi:10.1098/rstb.2008.0136

FlynN, E., \& Whiten, A. (2008a). Cultural transmission of tool use in young children: A diffusion chain study. Social Development, 17, 699-718. doi:10.1111/j.1467-9507.2007.00453.x

FlynN, E., \& Whiten, A. (2008b). Imitation of hierarchical structure versus component details of complex actions by 3- and 5-yearolds. Journal of Experimental Child Psychology, 101, 228-240. doi:10.1016/ j.jecp.2008.05.009

FranZ, M., \& NUNN, C. L. (2010). Investigating the impact of observation errors on the statistical performance of network-based diffusion analysis. Learning \& Behavior, 38, 235-242.

Giraldeau, L.-A., \& Lefebvre, L. (1987). Scrounging prevents cultural transmission of food-finding behavior in pigeons. Animal Behaviour, 35, 387-394.

Giraldeau, L.-A., \& Templeton, J. J. (1991). Food scrounging and 
diffusion of foraging skills in pigeons, Columba livia: The importance of tutor and observer rewards. Ethology, 89, 63-72.

Henrich, J., \& Gil-White, F. (2001). The evolution of prestige: Freely conferred deference as a mechanism for enhancing the benefits of cultural transmission. Evolution \& Human Behavior, 22, 165-196.

HILl, S. E., \& FLOM, R. (2007). 18- and 24-month-olds' discrimination of gender-consistent and inconsistent activities. Infant Behavior \& Development, 30, 168-173.

Hopper, L. M., FlynN, E. G., Wood, L. A. N., \& Whiten, A. (2010). Observational learning of tool use in children: Investigating cultural spread through diffusion chains and learning mechanisms through ghost displays. Journal of Experimental Child Psychology, 106, 8297. doi:10.1016/j.jecp.2009.12.001

Hopper, L. M., SPITERI, A., LAMBETH, S. P., SCHAPIRo, S. J., HORNER, V., \& Whiten, A. (2007). Experimental studies of traditions and underlying transmission processes in chimpanzees. Animal Behaviour, 73, 1021-1032.

Horner, V., \& Whiten, A. (2005). Causal knowledge and imitation/ emulation switching in chimpanzees (Pan troglodytes) and children (Homo sapiens). Animal Cognition, 8, 164-181. doi:10.1007/s10071 $-004-0239-6$

Horner, V., Whiten, A., Flynn, E., \& de WaAl, F. B. M. (2006). Faithful replication of foraging techniques along cultural transmission chains by chimpanzees and children. Proceedings of the National Academy of Sciences, 103, 13878-13883. doi:10.1073/ pnas.0606015103

Kendal, R. L., Custance, D. M., Kendal, J. R., Vale, G., Stoinski, T. S., Rakotomalala, N. L., \& Rasamimanana, H. (2010). Evidence for social learning in wild lemurs (Lemur catta). Learning \& Behavior, 38, 220-234.

Kirby, S., Cornish, H., \& SMith, K. (2008). Cumulative cultural evolution in the laboratory: An experimental approach to the origins of structure in human language. Proceedings of the National Academy of Sciences, 105, 10681-10686. doi:10.1073/pnas.0707835105

LALAND, K. N. (2004). Social learning strategies. Learning \& Behavior, 32, 4-14.

Lewis, C., Freeman, N. H., Kyriadidou, C., Maridaki-Kassotaki, K., \& BERRIDGe, D. M. (1996). Social influences on false belief access: Specific sibling influences or general apprenticeship? Child Development, 67, 2930-2947.

Lonsdorf, E. V., Eberly, L. E., \& Pusey, A. E. (2004). Sex differences in learning in chimpanzees. Nature, 428, 715-716.

Lyons, D. E., Young, A. G., \& KeIL, F. C. (2007). The hidden structure of overimitation. Proceedings of the National Academy of Sciences, 104, 19751-19756. doi:10.1073/pnas.0704452104

McGuigan, N., \& Graham, M. (in press). Cultural transmission of irrelevant tool actions in diffusion chains of 3- and 5-yearold children. European Journal of Developmental Psychology. doi:10.1080/17405620902858125

McGuigan, N., Whiten, A., Flynn, E., \& Horner, V. (2007). Imitation of causally opaque versus causally transparent tool use by 3- and 5-year-old children. Cognitive Development, 22, 353-364. doi:10.1016/j.cogdev.2007.01.001

Meltzoff, A. N. (1995). Understanding the intentions of others: Reenactment of intended acts by 18 -month-old children. Developmental Psychology, 31, 838-850.

Mesoudi, A., \& Whiten, A. (2008). The multiple roles of cultural transmission experiments in understanding human cultural evolution. Philosophical Transactions of the Royal Society B, 363, 3489-3501. doi:10.1098/rstb.2008.0129

NiElsen, M. (2006). Copying actions and copying outcomes: Social learning through the second year. Developmental Psychology, 42, 555-565. doi:10.1037/0012-1649.42.3.555

Plotkin, H. (2003). We-intentionality: An essential element in understanding human culture? Perspectives in Biology \& Medicine, 46, 283-296. doi:10.1353/pbm.2003.0028

Quinn, P. C., Uttley, L., Lee, K., Gibson, A., Smith, M., Slater, A. M., \& Pascalis, O. (2008). Infant preference for female faces occurs for same- but not other-race faces. Journal of Neuropsychology, 2, 15-26.

REAdER, S. M., \& Biro, D. (2010). Experimental identification of social learning in wild animals. Learning \& Behavior, 38, 265-283.

Richerson, P.-J., \& Boyd, R. (2005). Not by genes alone: How culture transformed human evolution. Chicago: University of Chicago Press.

Sandler, W., Meir, I., Padden, C., \& Aronoff, M. (2005). The emergence of grammar: Systematic structure in a new language. Proceedings of the National Academy of Sciences, 102, 2661-2665.

STERELNy, K. (2006). The evolution and evolvability of culture. Mind \& Language, 21, 137-165.

Thornton, A., \& Malapert, A. (2009). Experimental evidence for social transmission of food acquisition techniques in wild meerkats. Animal Behaviour, 78, 255-264.

Tomasello, M. (1999). The cultural origins of human cognition. Cambridge, MA: Harvard University Press.

Tomasello, M., Kruger, A. C., \& Ratner, H. H. (1993). Cultural learning. Behavioral \& Brain Sciences, 16, 495-552.

Whiten, A., Custance, D. M., Gomez, J.-C., Teixidor, P., \& Bard, K. A. (1996). Imitative learning of artificial fruit processing in children (Homo sapiens) and chimpanzees (Pan troglodytes). Journal of Comparative Psychology, 110, 3-14.

Whiten, A., \& FLYNN, E. (in press). The evolution of experimental "microcultures" in groups of young children: Investigating the process of transmission. Developmental Psychology.

Whiten, A., Horner, V., \& de WaAl, F. B. M. (2005). Conformity to cultural norms of tool use in chimpanzees. Nature, 437, 737-740.

Whiten, A., \& Mesoudi, A. (2008). Establishing an experimental science of culture: Animal social diffusion experiments. Philosophical Transactions of the Royal Society B, 363, 3477-3488. doi:10.1098/ rstb.2008.0134

Whiten, A., Spiteri, A., Horner, V., Bonnie, K. E., Lambeth, S. P., SchapIRo, S. J., \& DE WaAL, F. B. M. (2007). Transmission of multiple traditions between within and between chimpanzee groups. Current Biology, 17, 1038-1043.

Wood, L., KendaL, R., \& Flynn, E. (2010, July). Investigating indirect and direct transmission biases in children: Copying of irrelevant actions by five-year-old children is influenced by the age, but not task specific knowledge, of models. Poster presented at the Royal Society Meeting "Social Learning in Humans and Non-Human Animals: Theoretical and Empirical Dissections," Kavli Royal Society International Centre, Buckinghamshire.

(Manuscript received February 24, 2010; revision accepted for publication May 4, 2010.) 